Ospedaliera Universitaria Integrata Verona, Cardiology, Verona, Italy; ${ }^{3}$ Ospedale di Rovereto, Pediatrics, Rovereto (TN), Italy

Background: Acute Rheumatic Fever (ARF) is an immunomediated multisystem disease that occurs about 2-5 weeks after Group A Streptococcus Pyogenes beta-hemolytic (GAS) pharyngitis. After a negative peak in the 1980s, following the introduction of antibiotic prophylaxis, the disease is currently recovering. Rheumatic carditis is one of the most worrying aspects as it is still one of the major causes of cardiovascular death in the young-adult population. However, if diagnosed early and treated, sequelae with aortic and mitral valve involvement can be prevented.

Objectives: The aim of our study is a description of Acute Rheumatic Fever in all its manifestations in a cohort of pediatric patients belonging to the Azienda Ospedaliera Universitaria Integrata of Verona.

Methods: A retrospective analysis was conducted collecting all the cases of ARF, diagnosed by Jones's criteria, related to Pediatric Rheumatology and Pediatric Cardiology of Verona from January 2005 to December 2019. Demographic and clinical data were collected for all patients such as clinical presentation, disease evolution and cardiac involvement.

Results: 73 patients were analyzed, of whom 53 had an acute onset of ARF and 20 received a diagnosis of previous ARF due to indolent carditis. The prevalent age at the time of diagnosis in both groups was between 5 and 14 years of age. Among patients with acute onset, carditis was the most frequent major manifestation $(94.3 \%)$, followed by polyarthritis $(41.5 \%)$, chorea $(24.5 \%)$ and erythema marginatum (7.5\%). Only in one patient we could observe subcutaneous nodules (1.8\%). Regarding the minor manifestations, the increase in inflammation markers was present in $83 \%$ of cases and fever was present in $75.5 \%$, followed by arthralgia (58.4\%) and prolonged of PR interval to ECG $(9.4 \%)$. Carditis was also present in all 13 patients who presented chorea. Clinically, previously unknown heart murmur occurred in 28 patients. Therefore, the mismatch between cardiac objectivity and carditis finding is clear: infact, compared to an important finding of carditis (50 patients) only slightly more than half of the patients (28 patients) showed an evident clinic finding. Finally, no correlation was found between the levels of the antistreptolysin $\mathrm{O}$ titer and the severity of heart damage. Patients with early diagnosis of carditis were treated at onset with corticosteroid therapy according to the American Heart Association scheme and did not show valvular cardiac outcomes. A patient who received a late diagnosis of carditis currently presents a significant and permanent cardiac damage despite adequate steroid treatment undertaken at the time of diagnosis.

Conclusion: The description of this cohort of pediatric patients shows that the ARF has not disappeared in industrialized countries. Treatment of streptococcal infection (primary prophylaxis) plays a key role in preventing ARF. Of great impact is the prevalence of carditis which is present in $94.3 \%$ of patients. Early diagnosis is therefore of primary importance and the subsequent follow-up path, consisting of periodic therapy with penicillin (secondary prophylaxis) and periodic cardiological checks, greatly affects children's quality of life. Chorea, unlike what has been described in the literature, occurred simultaneously with the cardiac process, while the cutaneous manifestations (subcutaneous nodules and erythema marginatum), once pathognomonic of the rheumatic disease, are today of rare observation.

References:

[1] Carapetis, Beaton, Cunningham et al. Acute rheumatic fever and rheumatic heart disease. Nature Reviews Disease Primers. 2016;2:15084.

Disclosure of Interests: None declared

DOI: 10.1136/annrheumdis-2020-eular.4306

\section{THU0511 THE RISK FACTORS OF HIP OSTEOARTHRITIS IN JIA PATIENTS: THE DATA FROM A SINGLE CENTER RETROSPECTIVE CONTINUOUS STUDY}

L. Sorokina ${ }^{1,2}$, R. Raupov ${ }^{3}$, I. Avrusin ${ }^{3}$, M. Gharabaghtsyan ${ }^{3}$, N. Garipova ${ }^{1}$, S. Khrypov ${ }^{3}$, M. Dubko ${ }^{3}$, L. Snegireva ${ }^{3}$, T. Likhacheva ${ }^{3}$, A. Santimov ${ }^{3}$, E. Gaidar ${ }^{3}$, E. Isupova $^{3}$, I. Chikova ${ }^{3}$, T. Kornishina ${ }^{3}$, V. Masalova ${ }^{3}$, M. Kostik $^{1,3}$ ${ }^{1}$ Almazov National Medical Research Centre, Saint Petersburg, Russian Federation; 'Leningrad Regional Children's Hospital, Saint Petersburg, Russian Federation; ${ }^{3}$ Saint Petersburg State Pediatric Medical University, Saint Petersburg, Russian Federation

Background: Hip osteoarthritis (HOA) is a severe irreversible complication of patients with juvenile idiopathic arthritis (JIA) leads to intensive pain, disability and required total hip arthroplasty (THA) in childhood or in the young adults.

Objectives: the aim of our study was to evaluate risk factors of HOA in JIA patients.

Methods: we analyzed 753 patients with JIA for last 10 years. In each patient we observed if the HOA developed or no. We evaluated the main clinical JIA measurements, such a JIA category, CRP, ESR, WBC, PLT, active joints, initial or delayed hip involvement, ANA, HLA B27, onset age, JIA duration, time before
HOA and before THA, bone metabolic markers, treatment, particularly glucocorticosteroids (GCS), their cumulative doses, route of administration (oral, intra-articular, pulse therapy). HOA was confirmed with radiological assessment (MRI and CT). For comparison analysis used nonparamentric statistics. Each possible variable was evaluated with univariate regression analysis and further multiple regression analysis was applied.

Results: the comparative study between 2 groups shown in the table 1 . HOA was detected in $48(6.4 \%)$, in oligoarthritis- $0 \%$, in polyarthritis - $4.9 \%$, in psoriatic arthritis - 5.0\%, in enthesitis-related arthritis $-11.8 \%$, in systemic arthritis $19 \%$ $(p=0.0000001)$, THA was performed in $16(2.1 \%)$. Hip involvement at onset of JIA was similar in both groups $(49.5 \%$ vs $41.7 \%, p=0.367)$.

Table 1. Characteristics of JIA patients, depends on HOA development.

\begin{tabular}{lccc}
\hline Parameter & HOA $(\mathrm{n}=48)$ & No HOA $(\mathrm{n}=705)$ & $\mathrm{p}$ \\
\hline Onset age, y & $8.0(4.0 ; 12.5)$ & $5.9(3.0 ; 10.7)$ & 0.045 \\
HLA B27, $\mathrm{n}(\%)$ & $13 / 25(52.0)$ & $88 / 283(31.1)$ & 0.033 \\
JIA duration, y & $7.5(3.2 ; 12.1)$ & $4.2(1.8 ; 7.2)$ & 0.00007 \\
CRP, mgVI & $2.8(0.8 ; 20.9)$ & $1.3(0 ; 7.1)$ & 0.006 \\
Active joints, $\mathrm{n}$ & $11.0(6.0 ; 27.0)$ & $6.0(3.0 ; 12.0)$ & 0.000001 \\
Oral GCS, $\mathrm{n}(\%)$ & $21(43.8)$ & $131 / 704(18.6)$ & 0.00003 \\
GCS pulse-therapy, $\mathrm{n}(\%)$ & $21(43.8)$ & $114 / 702(16.2)$ & 0.000002 \\
GCS, cumulative doses, mg & $5000(3000 ; 16000)$ & $2000(1000 ; 4000)$ & 0.001 \\
Biologics, $\mathrm{n}(\%)$ & $43(89.6)$ & $308(43.7)$ & 0.0000001 \\
Remission, $\mathrm{n}(\%)$ & $24(50.0)$ & $461(65.4)$ & 0.03 \\
Time, before remission, y & $7.4(3.9 ; 12.3)$ & $3.0(1.4 ; 6.2)$ & 0.0000001 \\
Alkaline phosphatase, UVI & $129.3(84 ; 244)$ & $223(147 ; 386)$ & 0.00001 \\
Delayed hip involvement, $\mathrm{n}(\%)$ & $28(58.3)$ & $24 / 87(27.6)$ & 0.00004 \\
\hline
\end{tabular}

We calculated cut-off and OR of variables, associated with HOA development (table 2). After selection of 26 clinically meaningful and statistical significant risk factors only 22 pass the univariate regression analysis. In multiple regression analysis the main independent risk factors of HOA development were: HLA b27 $(p=0.001)$, oral GCS/pulse therapy GCS $(p=0.03)$ and alkaline phosphatase $\leq 165 \mathrm{U} / \mathrm{l}(\mathrm{p}=0.00006)$.

Table 2. Cutt-offs, odds ratio for HOA risk factors in JIA patients.

\begin{tabular}{lcccc}
\hline Parameter & Se & Sp & OR $(95 \% \mathrm{Cl})$ & $\mathrm{p}$ \\
\hline Active joints $>4$ & 89,6 & 42,0 & $6,2(2,4-15,9)$ & 0,000002 \\
Alkaline phosphatase $\leq 165 \mathrm{U} / \mathrm{l}$ & 65,9 & 70,8 & $4,7(2,4-9,2)$ & 0,000001 \\
GCS $>2700 \mathrm{mg}$ & 82,6 & 58,1 & $6,6(2,1-20,6)$ & 0,0004 \\
Time before remission $>5$ years & 66,7 & 78,9 & $7,5(3,1-18,0)$ & 0,0000001 \\
Time before biologics $>7,8$ years & 44,2 & 86,7 & $5,2(2,6-10,2)$ & 0,0000001 \\
\hline
\end{tabular}

Conclusion: the main risk factors of HOA were increased inflammatory activity, systemic arthritis, HLA B27, systemic corticosteroids, delayed remission and biologics, decreased bone metabolism. Delayed hip involvement underline the possible non-inflammatory mechanism of HOA, such as avascular necrosis.

Disclosure of Interests: None declared

DOI: 10.1136/annrheumdis-2020-eular.4632

\section{THU0512 HEALTH RISK BEHAVIORS IN ADOLESCENTS WITH JUVENILE IDIOPATHIC ARTHRITIS (JIA) IN THE COURSE OF DISEASE: RESULTS OF THE GERMAN MULTICENTER INCEPTION COHORT (ICON)}

F. Milatz ${ }^{1}$, I. Liedmann ${ }^{1}$, M. Niewerth ${ }^{1}$, J. Klotsche ${ }^{1,2}$, G. Horneff ${ }^{3,4}$, F. WellerHeinemann ${ }^{5}$, I. Foeldvari ${ }^{6}$, D. Windschall ${ }^{7}$, K. Minden ${ }^{1,2} .{ }^{1}$ German Rheumatism Research Centre, Berlin, Germany; ${ }^{2}$ Charité University Medicine, Berlin, Germany; ${ }^{3}$ Asklepios Klinik Sankt Augustin, Sankt Augustin, Germany; ${ }^{4}$ University Hospital Cologne, Cologne, Germany; ${ }^{5}$ Prof. Hess Children's Hospital, Bremen, Germany; ${ }^{6}$ Hamburg Centre for Pediatric and Adolescent Rheumatology, Hamburg, Germany; ${ }^{7}$ St.-Josef-Stift Nordwestdeutsches Rheumazentrum, Sendenhorst, Germany

Background: Health risk behaviors (HRB) are defined as specific forms of behavior associated with increased susceptibility to a specific disease or ill health on the basis of epidemiological or social data. The social implications of HRB include being able to use them as a way to gain respect and acceptance from peers, establishing independence from parents, or providing a subjective feeling of maturity and adequate stress management [1].

Objectives: Since chronically ill adolescents such as those suffering from JIA can develop a HRB that is challenging for optimal care the aim of this study was to compare the frequency of HRB in adolescents with JIA and their peers, and to determine whether they change during the course of disease.

Methods: Patients $\leq 16$ years of age with recently diagnosed JIA ( $<12$ months) were enrolled in the inception cohort of patients with newly diagnosed JIA (ICON), an ongoing prospective observational, controlled multicenter study 